\title{
THE CHIARI NETWORK AND THE VALVE OF THE INFERIOR VENA CAVA
}

BY

\author{
ELINOR D. U. POWELL AND JOAN M. MULLANEY \\ From the Departments of Medicine and Pathology, Trinity College, Dublin \\ Received January 27, 1960
}

The valve of the inferior vena cava is one of the most variable structures in the body, and has attracted sporadic interest for four centuries. Eustachius, writing in 1563, was the first to describe and classify its variations: he found that the valve was either partly replaced by interlacing fibres or consisted of a crescent of tissue or was minute. The valve was rediscovered by various authors for the next 300 years; and in the latter part of the nineteenth century, attention was focused on the more bizarre forms. Lauenstein (1876), when examining a hundred hearts, found five in which the valves of the inferior vena cava and the coronary sinus were formed from a continuous web of tissue; four of these valves were fenestrated.

Chiari described 11 cases in which the valve of the inferior vena cava, the Eustachian valve, was represented by networks with attachments to the superior vena cava and to the tubercle of Lower. While the Eustachian valve was usually derived only from the right venous valve, he claimed that networks with these widespread attachments were derived also from the embryonic septum spurium. He suggested that the network could act as a filter for thrombi that would otherwise affect the lung.

Several isolated instances of networks with these attachments have been reported since, and four more useful papers, in which a series of hearts has been examined, have also appeared. Yater (1929) found 11 large networks in the right atria of 120 hearts, and suggested that they could act as sources of thrombus formation. In a later paper (1956) he described how an embolus from the right femoral vein had become ensnared in such a network, and there were further emboli in the lung. Helwig (1932) found 8 large nets in 460 hearts, while Gresham (1957) found 4 in 260 adult hearts. Wright et al. (1948) have classified their findings rather differently and found either large fenestrated valves or valves with attached fibres in 5.6 per cent of 512 hearts. These latter authors and Yater did not describe the involvement of the septum spurium in all cases where there was a network, as did other writers including Chiari and Gresham: Helwig and Looser (1902) state that the networks Chiari reported in 1897 were derived only from the right venous valve. Owing to the wide variation in these structures, the classifications differ from paper to paper.

That networks associated with the valve of the inferior vena cava are quite common is not generally realized, partly perhaps because this part of the heart is frequently damaged when being removed from the chest at necropsy.

\section{Present Study}

We have examined 100 hearts that were removed with the inferior vena caval orifice intact from bodies undergoing routine necropsy. They were not in any way selected. In 85 of the cases clinical notes were available and electrocardiograms had been taken in 33 of them.

The following variations of the Eustachian valve were seen.

Group I. Three cases: neither ridge nor valve guarded the inferior vena caval orifice.

Group II. Eight cases: a ridge (the posterior part of the sinus septum) alone was present.

Group III. Nine cases: there was a small valve with no underlying ridge. 
Group IV. Eighty-three cases: both ridge and valve were present; in 24 of these the valve was either fenestrated or replaced, largely, by fibres.

These 24 cases have been further analysed according to their pattern and attachments, and all are illustrated by free-hand drawings as shown in Fig. 1.

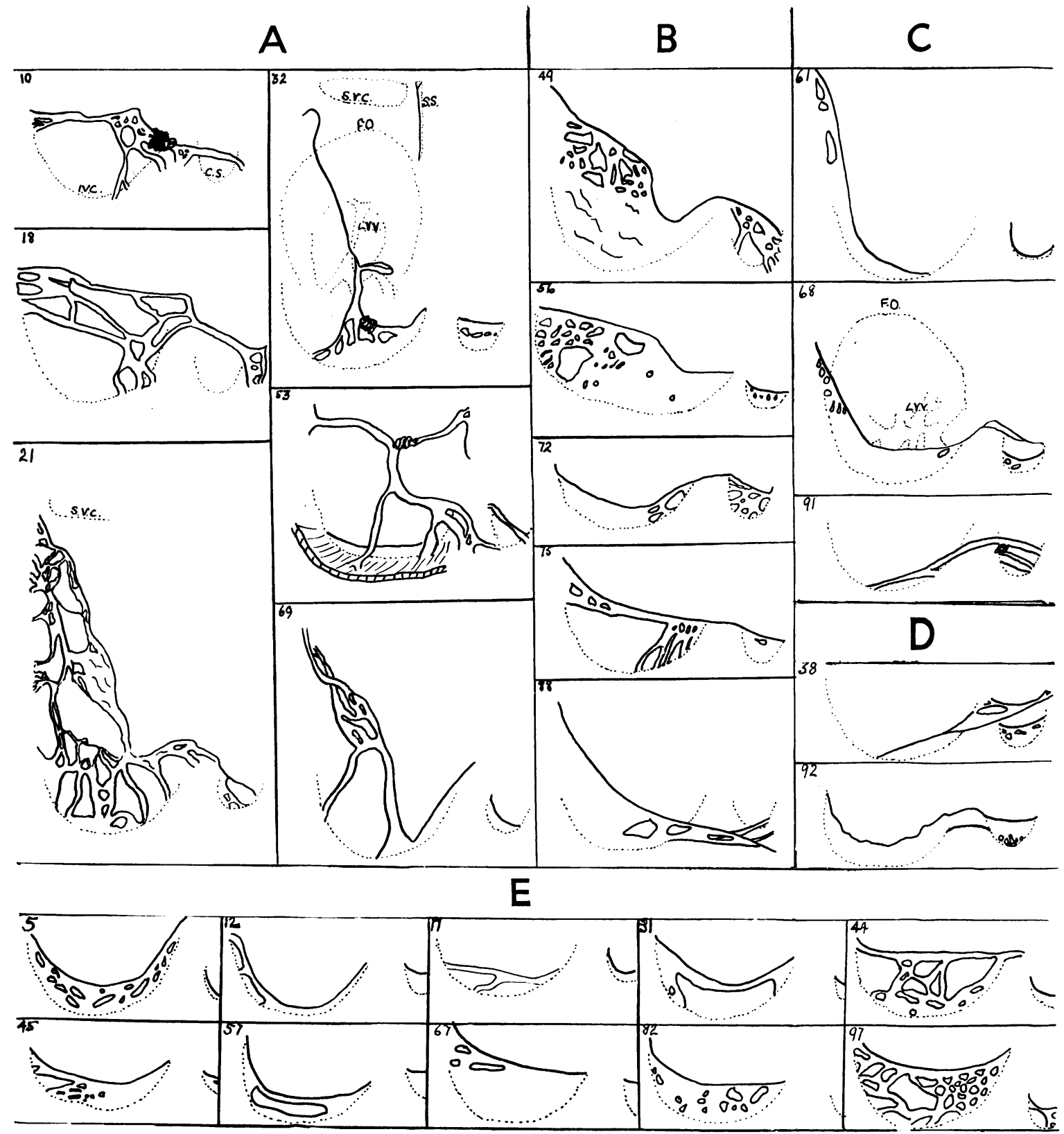

FIG. 1.-Diagrams of 26 Eustachian Valves. (A) Widespread networks made up of fibres. (B) Attachments beyond the caval orifice. (C) Small valves with wide attachments. (D) Other valves with common Eustachian and Thebesian valve. (E) Small, fenestrated valves.

S.V.C. = superior vena cava. $\quad$ S.S. =septum spurium. $\quad$ F.O. $=$ fossa ovalis.

L.V.V. = left venous valve. $\quad$ I.V.C. $=$ inferior vena cava 
In 10 cases the valve was fenestrated, but had no attachments beyond the caval orifice (Cases 5 , $12,17,31,44,45,57,67,82,97)$.

In 3 cases a small fenestrated valve was found with attachments beyond the caval orifice (Cases $61,68,91)$.

In 5 cases there were larger fenestrated valves, in two instances with convex upper borders, and with attachments beyond the caval orifice (Cases 49, 56, 72, 75, 88).

In 6 cases the valves were represented by fibres rather than fenestrated, with widespread attachments (Cases 10, 18, 21, 32, 53, 69).

In 12 cases there was continuity of tissue between the Eustachian and Thebesian valves (Cases $10,18,21,53,49,72,75,88,68,91,38,92)$.

In none of the hearts in this series was there connection between the valve of the inferior vena cava and the upper end of the crista terminalis, in front of the superior vena cava, as described by Looser, by Yater, and by Wright $e t$ al. In several instances (including Case 32) an isolated crest of tissue projecting about $2 \mathrm{~mm}$. into the right atrium ran down from this part of the crista terminalis to the septal wall. Fenestrations of the Thebesian or coronary valve were even more common, occurring in a total of 37 cases.

The proportion of 24 cases out of 100 having fenestration of the Eustachian valve agrees closely with that of Gresham, who reports a proportion of 25 per cent, but is much higher than in the series of Wright et al., in which only 7 per cent showed fenestration of this valve.

The question of thrombus formation, or delay, has been raised by several authors, as mentioned earlier. It would seem likely that the valve fenestrated by small holes might act as a sieve for the filtering of emboli, although in those valves with redundant tissue such as Cases 21 (Fig. 2),

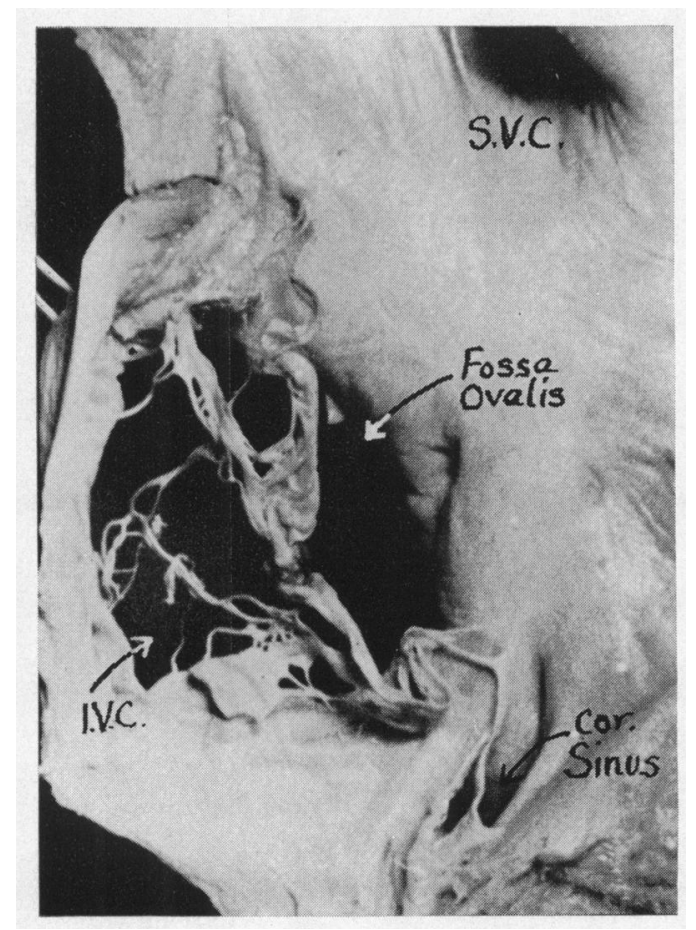

Fig. 2.-Chiari network at inferior vena cava. Note continuity of tissue between the Eustachian and Thebesian valves. 
49, and 56, the embolus might be shaken off as the valve moved in the atrial currents. There was no evidence of ante-mortem thrombosis adherent to any of this type of valve, nor were there pulmonary infarcts. When the valve was largely represented by fibres, sometimes whorled or knotted, thrombus formation was to be expected, and amongst the fibres of two such cases (10 and 53) firm, adherent ante-mortem thrombi were found. Case 10 had had atrial fibrillation for six months before her death at 80 years of age, and the network was the only site of atrial thrombosis. The fibres in Case 53 (Fig. 3) were so tightly stretched across the atrial cavity as to act as

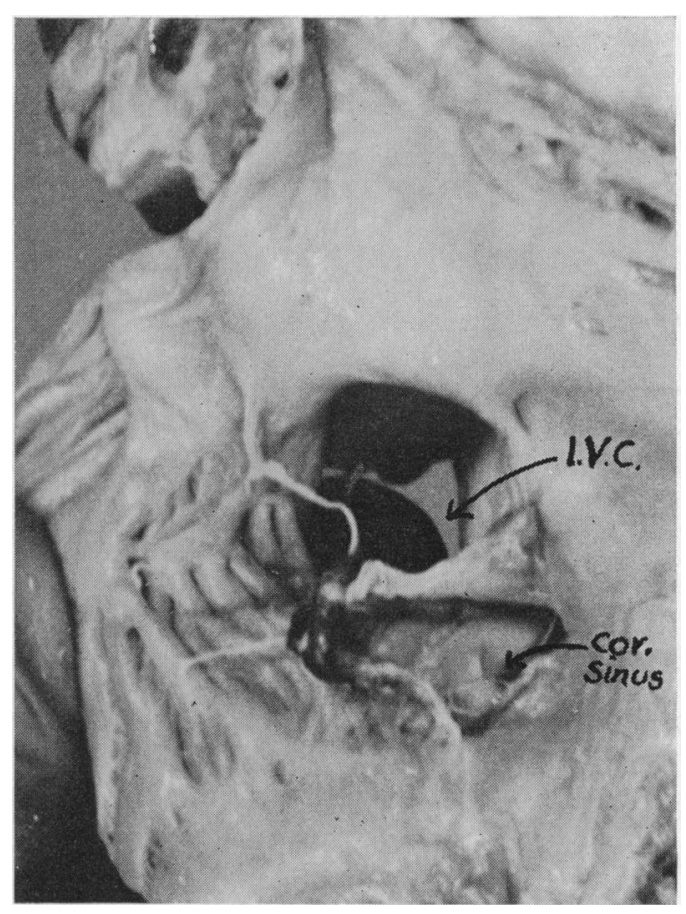

FIG. 3.-Chiari network with ante-mortem thrombus enmeshed and with coiling of fibres. There is continuity of tissue between the Eustachian and Thebesian valves.

an obstruction to the flow of blood, but there were no symptoms of such obstruction in the patient before death. In three of our series (Cases 10, 32, and 53) it seemed that the fibres of the network were torn during life. In each case fibres were whorled round another strand of the network. This was the site of thrombus formation in Case 10, and in Case 32 an additional free loop hung from the middle of the longest strand. It appears possible that such strands could break free during life, although being so fine it is unlikely that they would cause symptoms when lodging in a small branch of the pulmonary artery. Fibres of the Thebesian valve were the site of thrombus formation in eight cases.

Gresham, with support from Jordan, has suggested that the tissue of the network may conduct the atrial impulse. When the network stretches superiorly as far as the superior vena cava, it passes close to the region of the sinu-atrial node described by Keith and Flack (1907). In two fœtuses, 16 and $21 \mathrm{~cm}$. long, examined by Oppenheimer and Oppenheimer (1912) the node was found in close relation to the base of the venous valve, giving embryological support to Gresham's hypothesis. The latter has demonstrated muscle and nerve tissue in some of the networks he describes. The finding of a varying $\mathbf{P}-\mathbf{R}$ interval in the electrocardiogram of one of his cases is 
probably incidental; at any rate, its significance is difficult to assess as he does not state the nature of the irregularity nor its duration. Electrocardiograms taken in four patients in our series (Cases $21,49,53,61)$ with networks having attachments near the superior vena cava showed each a constant $\mathbf{P}-\mathbf{R}$ interval.

In order to follow the discussion of the embryological origin of the networks of the right atrium by Wright et al. (1948) and earlier writers, it is necessary to trace the sites of the primitive valves in the adult heart. Tandler (1912) and Odgers (1935) have illustrated the valves between the sinus venosus and the atrium in the embryo of $5-10 \mathrm{~mm}$. The venous valves, of which the right is the larger, encircle this orifice and project into the atrial cavity. They are united superiorly by a crest of tissue, the septum spurium, which runs forward at right angles from this attachment, under the atrial roof, projecting for a variable distance into the atrial cavity. At $9 \mathrm{~mm}$. the septum spurium is deep, but then starts to regress in favour of the right venous valve. This latter valve in embryos from 13-30 mm. in length is so deep as to project across two-thirds of the atrial cavity (Streeter, 1948; Licata, 1954). Later, by the appearance and coalescence of fenestræ this valve regresses. The superior vena cava in the earliest embryos in which it can be identified opens into the right horn of the sinus venosus. This horn is then taken up into the main part of the sinus venosus, which lies then directly behind the right atrium. At this stage the regressing septum spurium forms a thin crest anterior to the superior vena cava $(25-30 \mathrm{~mm}$. embryo). The right venous valve extends from this position downwards, with its base attached first to the lateral margin of the superior vena cava, and then to the postero-lateral wall of the atrium, before bounding the orifices of the inferior vena cava and coronary sinus. Anteriorly, in a $50-\mathrm{mm}$. embryo of our own the valve extended to the atrial septum, as does the anterior strand of Case 53, who died at the age of 45 years. Usually the upper part of the valve regresses completely, leaving narrow and unconnected Eustachian and Thebesian valves.

The left venous valve, bounding the medial wall of the sinus venosus, becomes attached to the atrial septum. Remnants of this valve were seen in three hearts (Cases 32 and 68; also Case 15, not illustrated): in these there were trabeculæ at the base of the foramen ovale.

It appears then that only those networks with attachments anterior to the superior vena cava can be said to be derived in part from the septum spurium. Few authors make this distinction when describing these networks and their illustrations do not show clearly the extent of the network attachments. Although networks of the Eustachian valve are quite common, those with such widespread attachments are very rare; Wright et al. (1948) found only one case in their series of 512 hearts, and there is no such network in our series. The isolated crest of tissue running from the upper end of the crista terminalis on to the atrial wall, seen in the heart of Case 32 and in several other hearts, is probably a remnant of the septum spurium. Chiari's name is used generally for the widespread nets to which he first drew attention and it should be retained, although few of these are derived in part from the septum spurium as he had supposed.

In conclusion, it appears that these interesting networks are quite common. When reduced to narrow fibres they may be sites for thrombus formation, especially when there is atrial fibrillation. The fibres of the network are sometimes torn during life, and may break free. The fenestrated types may rarely remove emboli from the circulation, but this is purely by chance, and further emboli are likely to reach the lung. The Eustachian and Thebesian valves should be examined routinely by pathologists at autopsy, care being taken to open the right atrium by an antero-lateral cut.

\section{SUMmaRY}

The variation of the Eustachian valve in 100 routine autopsies is discussed and instances are given in which the valve has been the source of thrombus formation. In other instances the valve had become torn during life. Various reports on this subject are reviewed and the embryology is discussed. 


\section{REFERENCES}

Chiari, H. (1897). Beittr. path. Anat., $22,1$.

Eustachius, B. (1563). Opuscula Anatomica.

Evangelisti, T. (1940). Arch. Vecchi per anat path. med. clin., 2, 895.

Franklin, K. J. (1948). Cardiovascular Studies. Blackwell, Oxford.

Gresham, G. A. (1957). Brit. Heart J. 19, 381.

Helwig, F. C. (1932). Amer. J. Path., 8, 73.

Jordan, W. R. (1926). Arch. Path., 2, 840.

Licata, R. H. (1954). Amer. J. Anat., 94, 73.

Lauenstein, C. (1876). Virchows Archiv. path. Anat., 68, 632.

Looser, E. (1902). Quoted by Yater (1929).

Odgers, P. N. R. (1935). J. Anat., 69, 412.

Oppenheimer, A., and Oppenheimer, B. S. (1912). Anat. Rec., 6, 487.

Patten, B. M. (1953). Human Embryology, 2nd ed. Churchill, London.

Streeter, G. L. (1948). Contribution to Embryology 32. Carnegie Inst., Wash.

Tandler, in Mall, F. P. (1912). Human Embryology. Lippincott \& Co., Philadelphia and London.

Wilson, R. (1938). J. Amer. med. Ass., 111, 917.

Wright, R. R., Anson, B. J., and Cleveland, H. C. (1948). Anat. Rec., 100, 331.

Yater, W. M. (1929). Arch. Path., 7, 418.

- (1956). Amer. Heart J., 11, 542. 